\title{
Réajustements temporels et syllabiques après l'effacement optionnel du schwa en français
}

\author{
Beltzung, Jean-Marc \& Wallet, Lucille \\ 1 \\ Laboratoire de linguistique de Nantes (EA 3827, Université de Nantes) \\ jean-marc.beltzung@univ-nantes.fr \\ 2 \\ Laboratoire de phonétique et phonologie (UMR 7018, CNRS/Sorbonne-Nouvelle) \\ lucille.wallet@univ-paris3.fr
}

\section{Introduction}

En français moderne standard, le schwa peut s'effacer dans différentes positions (cf. Lebel 1968, Anderson 1982, Dell 1973, Rialland 1986, Tranel 1995, Fougeron \& Steriade 1997, 1999, Steriade 2000, Barns \& Kavitskaya 2003, entre autres). L'une des hypothèses sans doute la plus forte concernant l'effacement du schwa a été émise par Rialland (1986). Selon elle, même si le schwa est effacé dans les formes de surface, la consonne précédente n'est pas resyllabée et le noyau projeté par le schwa est conservé par la propagation de la consonne suivante sur la position vocalique si celle-ci est une sonante (cf. /də\#воl/ $\rightarrow$ [dқ̣.коl] « de rôle » vs. /dьol/ $\rightarrow$ [dкоl] « drôle ») ou par la non association de matériel segmental sur la position vocalique si la consonne suivante est une obstruante. Fougeron \& Steriade (1997), qui ont également étudié les effets de l'effacement optionnel du schwa, notamment d'un point de vue articulatoire (mesures du contact linguopalatal), montrent que l'effacement de ce dernier laisse effectivement une trace qui peut être masquée par les consonnes adjacentes. Selon elles, les comparaisons entre des clusters consonantiques qui dérivent de l'effacement du schwa et les clusters consonantiques lexicaux (cf. d'rôle « de rôle » vs. drôle) montrent que les propriétés qui distinguent ces deux types de clusters proviennent de fines caractéristiques articulatoires contenues dans les formes sous-jacentes, lesquelles seraient maintenues dans les formes de surface après l'effacement du schwa. Notons également que Lebel (1968), qui avait comparé la durée des consonnes dans des clusters lexicaux et des clusters dérivés de l'effacement du schwa chez trois locuteurs, avait mis en évidence que l'effacement de ce dernier provoquait un allongement compensatoire de la consonne précédente.

Cet article traite des réajustements syllabiques et temporels qui découlent de l'effacement optionnel du schwa dans le démonstratif français /sə/ «ce» devant un mot commençant par une occlusive labiale ou coronale non voisée (i.e. /p/ et /t/). Dans un cadre linéaire de la phonologie, cet effacement optionnel peut être formalisé par la règle suivante :

$$
\text { Effacement optionnel du schwa }
$$$$
\text { ə } \rightarrow \varnothing /(\mathrm{V}) \# \mathrm{C}
$$

En d'autres termes, cette règle prédit que le schwa français peut s'effacer dans un morphème monosyllabique de type \#Cə\# devant un morphème à initiale consonantique. D'un point de vue syllabique, l'application de cette règle sur le morphème démonstratif /sa/ peut théoriquement provoquer certains réajustements : (i) la fricative coronale peut être syllabée dans l'attaque de la syllabe suivante, créant des clusters derivés de type [\#sp] et [\#st] qui existent par ailleurs dans certains mots lexicaux ; (ii) la fricative coronale peut être syllabée dans la coda de la syllabe précédente, créant des séquences hétérosyllabiques de type [s.t] et [s.p], (iii) la fricative coronale peut être à la fois syllabée en coda de la syllabe précédente et en attaque de la syllabe suivante, créant des séquences ambisyllabiques ou géminées de type [s.st] et [s.sp] et (iv) la fricative peut constituer une syllabe en elle même et être réalisée comme une consonne syllabique de type [s.t.]. 
L'hypothèse que nous explorons ici est que les séquences $\mathrm{s}+\mathrm{C}$ qui proviennent de l'effacement optionnel $\mathrm{du}$ schwa, bien qu'homophones aux séquences $\mathrm{s}+\mathrm{C}$ lexicales dans les formes de surface, subissent un réajustement syllabique et/ou temporel qui se traduit par l'allongement de la fricative coronale non voisée précédente. Le but principal de ce processus consisterait à maintenir un contraste entre les clusters dérivés de l'effacement du schwa et les clusters lexicaux dans les formes de surface. Néanmoins, cette hypothèse n'exclut pas le fait que certains réajustements temporels indépendants se manifestent, notamment sur les occlusives.

\section{Méthodologie}

\subsection{Corpus}

Afin de tester notre hypothèse, un corpus basé sur six triplés de phrases syntaxiquement identiques a été constitué. Chaque triplé est composé (i) d'une phrase contenant le démonstratif /sə/ suivi par un mot débutant par une occlusive labiale ou coronale, (ii) de la même phrase où le schwa a été effacé (s\#\{p,t\}) et (iii) d'une phrase contenant un cluster lexical en position initiale de mot $(/ \# \mathrm{~s}\{\mathrm{p}, \mathrm{t}\} /)$ sans démonstratif à sa gauche. Le tableau 1 ci-dessous présente les six triplés sélectionnés qui composent le corpus (où ['] note l'effacement du schwa) :

$\mathrm{s}(\partial)+$ Labial

1. L'entrée dans ce port est gratuite

L'entrée dans c'port est gratuite

La chaîne de sport est gratuite

2. La trace de ce pas est mystérieuse

La trace de c'pas est mystérieuse

La salle de spa est agréable

3. Le lait de ce pis est mauvais

Le lait de c'pis est mauvais

La toile de spi est marron $\mathrm{s}(\mathrm{\partial})+$ Coronal

4. Le son de ce tar est magique

Le son de c'tar est magique

La vie de star est facile

5. Le beau-frère de ce type est velu

Le beau-frère de c'type est velu

Le sommet du stipe est velu

6. La difficulté de ce thème est d'éviter les fautes

La difficulté de c' thème est d'éviter les fautes

La difficulté d'un stemm est d'éviter les chutes

Tableau 1. Triplés mettant en évidence les différentes structures étudiées.

Il est important de noter que le nombre de syllabes ainsi que la position syllabique des clusters étudiés ont été contrôlé dans chaque triplé. Néanmoins, seule la fréquence lexicale et le débit de parole n'ont pu être contrôlés, ce qui peut entraîner de la variabilité intra-individuelle.

\subsection{Recueil de données et segmentation}

Ce travail est basé sur les enregistrements de dix sujets francophones - soit 5 hommes et 5 femmes - âgés de 27 à 54 ans.

Les différentes prises de données ont eu lieu dans une pièce acoustiquement calme de l'Institut de Linguistique et Phonétique Générale Appliquées (Paris III). Les sujets étaient assis de manière confortable et dans une position verticale afin de faciliter la phonation. Ceux-ci portaient un micro-casque de type AKGC520 orienté à $45^{\circ}$ de la bouche (soit 3-4 cm des lèvres), relié à une carte son EDIROL FA 66 dont la fréquence d'échantillonnage était réglée à $44100 \mathrm{~Hz}$ (soit 16 bits). Cette carte était directement reliée à un PC portable afin d'obtenir des enregistrements de bonne qualité. Les sujets avaient pour 
consigne de lire le protocole à un rythme régulier et de répéter les phrases accrochées ou sur lesquelles ils bloquaient. De manière générale, notre analyse se base sur deux lectures fluides du corpus par sujets, présenté dans un ordre aléatoire $(n=360)$. Les prises de données ont été effectuées à l'aide du logiciel Praat (Boersma \& Weenink 1996) et le traitement statistique avec le logiciel R.

La segmentation des données a été faite manuellement en manipulant conjointement les spectrogrammes et les oscillogrammes. Les segments ont été écoutés attentivement et la réalité perceptive a été associée à la réalité acoustique. Le début des occlusives a été segmenté (i) en repérant la fin du F2 de la voyelle précédente qui concorde avec la phase de silence dans le cas de la structure avec démonstratif pleinement réalisé ou (ii) en repérant la fin du bruit de friction de la fricative précédente dans le deux autres cas. La fin des occlusives a été segmentée en repérant l'apparition du F2 de la voyelle suivante. En ce qui concerne la fricative coronale, celle-ci a été segmentée en se basant sur le début et la fin du bruit de friction figurant sur les spectrogrammes ainsi que sur les changements de formes du signal. A partir de cette segmentation, la durée des consonnes a été extraite automatiquement à l'aide d'un script Praat réalisé à cet effet.

\section{Résultats}

\subsection{Tests préalables}

Tests d'homoscédasticité des variances. Le test de Brown-Forsythe, un type de test de Levene basé non pas sur la moyenne mais sur la médiane, a été pratiqué sur l'ensemble des données afin de vérifier l'homogénéité des variances. Celui-ci montre que les deux variables dépendantes dont il est question ici, durée-C et durée-S, ont des variances similaires $(\mathrm{p}=.615$ et $\mathrm{p}=.351$ respectivement) et peuvent par conséquent être comparées.

Tests de normalité. Afin de vérifier la normalité des distributions, le test de Lilliefors a été pratiqué sur l'ensemble des données. Si celui-ci montre que la durée de la fricative suit une distribution normale, $\mathrm{p}=$ .096 , il n'en est pas de même concernant la durée des occlusives, $p=.002$. Cette non-normalité des données peut poser un problème si nous désirons appliquer un test paramétrique tel qu'une ANOVA.

Transformation logarithmique. L'application d'un test paramétrique nécessite la normalisation de la durée des occlusives. Pour ce faire, chaque observation a été transformée en échelle logarithmique de base-e (logarithme naturel ou népérien). Une fois ces mesures normalisées, l'application du test de Lilliefors montre que les observations sont, in fine, normalement distribuées $(\mathrm{p}=.591)$.

\subsection{Analyses statistiques}

\subsubsection{Durée de l'occlusive}

Afin de vérifier l'hypothèse selon laquelle la durée de la consonne qui suit la fricative coronale [s] est identique quels que soient la structure morphologique $(\# \mathrm{C}, \mathrm{s} \# \mathrm{C}$ et $\# \mathrm{sC})$ et le lieu d'articulation (labiale et coronale) de la consonne (H0), une ANOVA à deux facteurs $(3 \times 2)$ a été conduite sur l'ensemble des données normalisées. Celle-ci montre un effet significatif de la structure morphologique et du lieu d'articulation sur la durée de la consonne, $F(2,354)=9.02, \mathrm{p}<.001, \eta^{2}=.05$ et $F(1,354)=5.22, \mathrm{p}=.02$, $\eta=.01$ respectivement.

Les analyses post-hoc issues du test HSD (Honestly Significant Difference) de Tukey montrent qu'il existe un effet significatif de la structure morphologique sur la durée de l'occlusive entre, d'une part, la structure \#C $(M=115.31, S D=31.21)$ et la structure \#sC $(M=100.27, S D=24.94), \mathrm{p}<.001$ et, d'autre part, la structure \#C $(M=115.31, S D=31.21)$ et la structure $\mathrm{s} \# \mathrm{C}(M=102.36, S D=25.21), \mathrm{p}=.003$, mais pas entre la structure $\mathrm{s} \# \mathrm{C}$ et la structure $\# \mathrm{sC}, \mathrm{p}=.771$. Ainsi, la durée de l'occlusive en attaque 
simple (\#C) est en moyenne plus longue de $15.03 \mathrm{~ms}(95 \% \mathrm{CI}:$ 6.81-23.26) que la durée de l'occlusive en attaque complexe $(\# \mathrm{SC})$. De même, la durée de l'occlusive en attaque simple (\#C) est en moyenne plus longue de $12.95 \mathrm{~ms}$ (95\% CI: 4.72-21.18) que l'occlusive qui se trouve dans des structures de type s\#C.

Enfin, le test montre un effet significatif du lieu d'articulation sur la durée des occlusives $(p=.023)$. La durée des occlusives labiales $(M=108.91, S D=26.93)$ est en moyenne plus longue de $5.85 \mathrm{~ms}(95 \% \mathrm{CI}$ : .24-11.46) que celle des occlusives coronales $(M=103.06, S D=28.82)$. Néanmoins, l'analyse post-hoc montre qu'il n'existe aucune interaction significative de la position et du lieu d'articulation sur la durée des occlusives entre les structures $\mathrm{s} \# \mathrm{C}$ et \#sC.

Les graphiques en barres et les boîtes à moustaches de la figure 1 montrent la distribution de la durée moyenne des occlusives en fonction de la structure morphologique et du lieu d'articulation des consonnes.
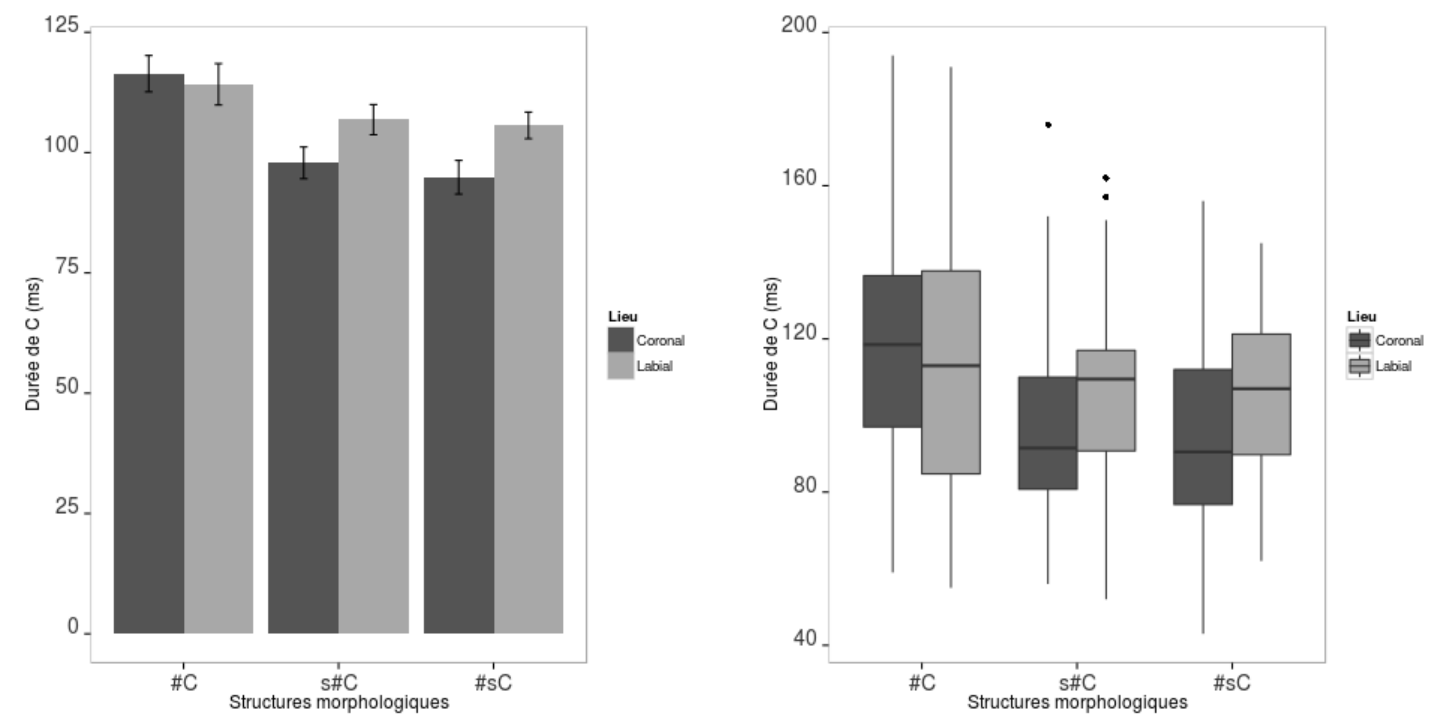

Graphique 1. Durée moyenne (en ms.) de l'occlusive orale en fonction de la structure morphologique et du lieu d'articulation.

\subsubsection{Durée de la fricative coronale}

Afin de vérifier l'hypothèse selon laquelle la durée de la fricative coronale [s] est identique quelles que soient la structure morphologique et le lieu d'articulation de la consonne suivante (H0), une ANOVA à deux facteurs $(3 \times 2)$ a été menée sur l'ensemble des données. Celle-ci montre un effet significatif de la structure morphologique et du lieu d'articulation de l'occlusive subséquente sur la durée de la fricative, $F(2,354)=24.38, \mathrm{p}<.001, \eta^{2}=.12$ et $F(1,354)=26.48, \mathrm{p}<0.001, \eta^{2}=.06$ respectivement.

Les analyses post-hoc issues du test HSD de Tukey montrent qu'il existe un effet significatif de la structure morphologique sur la durée de la fricative entre chacune des paires comparées. Ainsi, la durée de la fricative de la structure \#sə $(M=107, S D=21.14)$ est en moyenne plus longue de $18.35 \mathrm{~ms}(95 \% \mathrm{CI}$ : 12.16-24.54) que celle de la structure $\#_{\mathrm{SC}}(M=88.66, S D=20.08), \mathrm{p}<.001$. De même, la durée de la fricative de la structure $\mathrm{s} \# \mathrm{C}(M=98.63, S D=21.96)$ est en moyenne plus longue de $9.98 \mathrm{~ms}(95 \% \mathrm{CI}$ : 3.78-16.17) que celle de la structure \#sC, $\mathrm{p}<.001$. Enfin, la durée de la fricative de la structure \#sə est en moyenne plus longue de $8.38 \mathrm{~ms}(95 \% \mathrm{CI}: 2.18-14.57)$ que celle de la structure $\mathrm{s} \# \mathrm{C}, \mathrm{p}=.004$. 
Le test montre également un effet significatif du lieu d'articulation de l'occlusive sur la durée du [s]. Ainsi, la durée de la fricative est en moyenne plus longue de $11.06 \mathrm{~ms}$ (95\% CI : 6.83-15.28) devant une labiale $(M=103.63, S D=23.57)$ que devant une coronale $(M=92.57, S D=19.53)$. Néanmoins, le test post-hoc montre, de manière attendue, que le lieu d'articulation de l'occlusive n'a aucun effet significatif sur la durée de la fricative dans les structures \#sə, $\mathrm{p}=.23$. Dans ce qui va suivre, nous considérons uniquement les interactions de la structure morphologique et du lieu d'articulation de l'occlusive sur la durée de la fricative dans les structures de type $\mathrm{s} \# \mathrm{C}$ et \#sC, qui nous intéressent plus particulièrement.

Les comparaisons du test de Tukey mettent en évidence que seules deux paires, $\mathrm{s} \# \mathrm{t} v$. \#st et \#sp vs. s\#t respectivement, présentent des interactions non significatives. Ainsi, la durée de [s] dans la structure s\#t est en moyenne plus longue de $8.80 \mathrm{~ms}(95 \% \mathrm{CI}:-1.86-19.46)$ que celle de la structure \#st sans pour autant que cette différence soit significative, $\mathrm{p}=.171$. De même, la durée de [s] dans la structure \#sp est en moyenne plus longue de $2.48 \mathrm{~ms}(95 \% \mathrm{CI}:-8.18-13.15)$ que celle de la structure s\#t sans pour autant que cette différence soit significative, $p=.99$. En ce qui concerne les autres paires, celles-ci présentent toutes une interaction significative. Ainsi, la durée de la fricative de la structure \#sp est en moyenne plus longue de $11.28 \mathrm{~ms}(95 \% \mathrm{CI}: 0.62-21.95)$ que celle de la structure \#st, $\mathrm{p}=.003$. De même, la durée de la fricative de la structure s\#p est en moyenne plus longue de $22.43 \mathrm{~ms}$ (95\% CI : 11.77-33.09) que celle de la structure \#st, $\mathrm{p}<.001$. La durée du [s] de la structure $\mathrm{s} \# \mathrm{p}$ est en moyenne plus longue de $13.63 \mathrm{~ms}$ (95\% CI : 2.97-24.30) que celle de la structure s\#t, $\mathrm{p}=.003$. Enfin, la durée de la fricative de la structure s\#p est en moyenne plus longue de $11.15 \mathrm{~ms}$ (95\% CI : 0.49-21.81) que celle de la structure \#sp, p= .003 .

Les graphiques en barres et les boîtes à moustaches de la figure 2 montrent la distribution de la durée moyenne de la fricative [s] en fonction de la structure morphologique et du lieu d'articulation des consonnes.
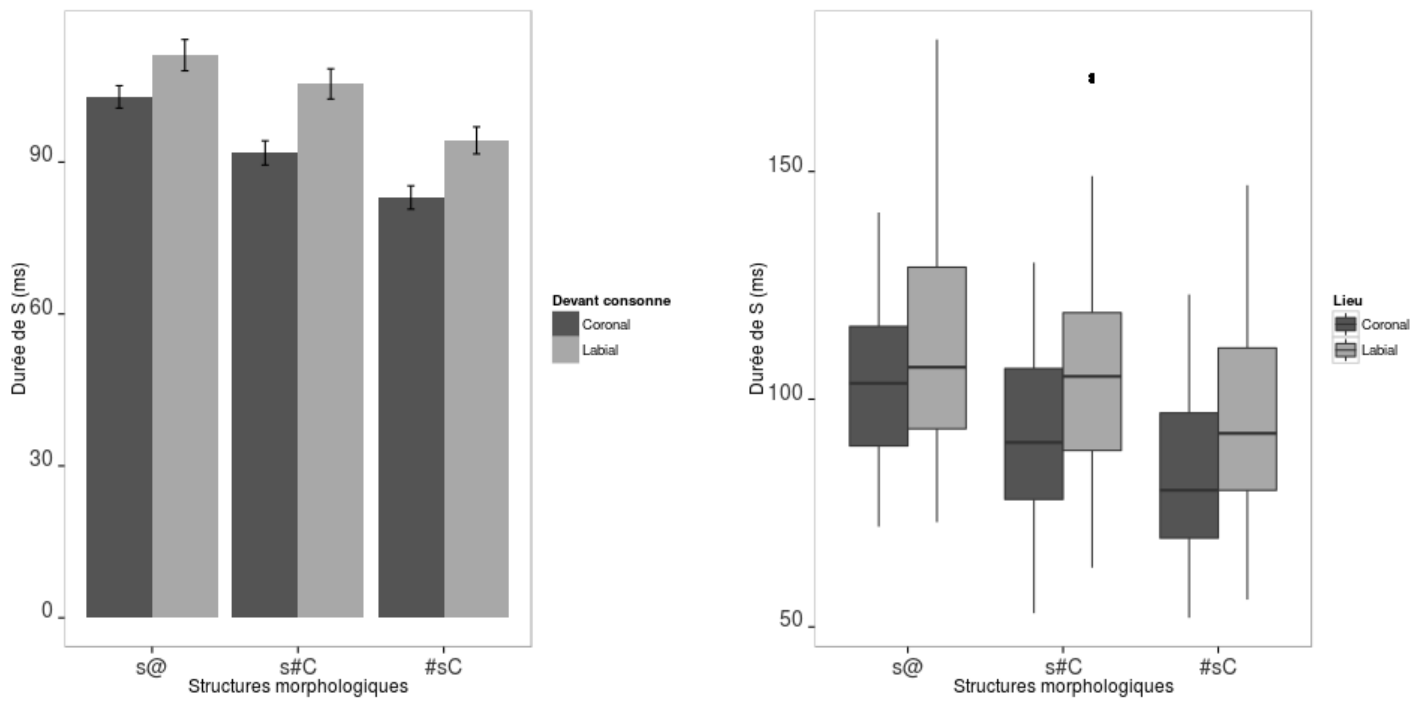

Graphique 2. Durée moyenne (en ms.) de la fricative coronale [s] en fonction de la structure morphologique et du lieu d'articulation de la consonne qui suit (où @ représente le schwa). 


\section{Discussion et analyse}

\subsection{Interprétation des tests statistiques}

D'un point de vue statistique, les résultats mettent en évidence un certain nombre d'effets intéressants liés à l'effacement optionnel du schwa. Premièrement, le fait que la durée des occlusives des formes $\mathrm{S \# C}$ et \# $\mathrm{sC}$ ne soit pas statistiquement différente, et ce quel que soit le lieu d'articulation de ces dernières, suggère que, contrairement aux formes $\# \mathrm{C}$ devant lesquelles schwa est pleinement réalisé ${ }^{1}$, ces deux types de clusters partagent la même structure segmentale. En d'autres termes, l'agencement linéaire des segments est identique dans les formes de surface puisqu'une fricative coronale précède une occlusive labiale ou coronale dans les deux cas (homophonie). De plus, les résultats confirment le fait que la phase de tenue des occlusives labiales est plus longue que celle des occlusives coronaIes et vélaires (Klatt 1976, Zue 1976, O'Shaughnessy 1981, Byrd 1993 entre autres). Ceci s'explique par un effet aérodynamique lié à la pression intraorale (PIO). En effet, la constriction en avant du conduit oral des occlusives labiales implique une augmentation plus lente de la PIO (cf. Byrd 1993) et, in extenso, de la durée de la tenue de l'occlusion consonantique ${ }^{2}$. Deuxièmement, les résultats mettent en évidence une hiérarchie de la durée de la fricative [s] principalement déterminée par la position prosodique (\#sə $>\mathrm{s} \# \mathrm{C}>\# \mathrm{sC}$ ). Il paraît évident que la durée de la fricative soit plus longue lorsqu'elle occupe une position d'attaque simple. En effet, O'Shaughnessy (1981:404) note que les fricatives en attaque simple sont plus longues de $19 \%$ que les fricatives en attaque complexe. Selon nous, le fait que la durée du [s] des formes de type $\mathrm{s} \# \mathrm{C}$ soit statistiquement plus longue - environ $10 \mathrm{~ms}$ en moyenne - que celle des formes \#sC pourrait refléter une différence dans l'agencement prosodique de ces séquences (hétérosyllabicité vs. homosyllabicité). Troisièmement, il semble que le lieu d'articulation de l'occlusive ait un effet sur la durée de la fricative précédente dans les clusters $\mathrm{s} \# \mathrm{C}$ et $\# \mathrm{sC}$. Ainsi, le fait que la fricative [s] soit statistiquement plus longue environ $11 \mathrm{~ms}$ en moyenne - devant une labiale peut s'expliquer par une différence de composante segmentale. En effet, si nous raisonnons en termes de primitives phonologiques unaires (cf. la théorie des éléments, Kaye et. al 1990, Harris \& Lindsey 1995, Scheer 1998, Nasukawa \& Backley 2008, entre autres), la fricative /s/ possède les éléments de résonance $|\underline{\mathrm{AI}}|$ (alvéolaire) alors que l'occlusive labiale ne possède que l'élément $|\underline{\mathrm{U}}|$ (labialité). Le fait que ces deux segments ne partagent pas le même lieu d'articulation (i.e. les mêmes éléments) implique par conséquent une implémentation phonétique séquentielle de leurs éléments, ce qui provoque une augmentation des durées segmentales que nous ne remarquons pas, de manière significative, dans les séquences homorganiques de type s\#t et \#st (coronalcoronal).

En outre, ces résultats montrent que l'hypothèse de Stériade (2000), qui stipule que l'effacement optionnel du schwa n'est jamais compensée dans les formes de surface (cf. la notion de PARADIGM UNIFORMITY) peut être remise en question. Selon elle, la durée segmentale des consonnes adjacentes au schwa qui sont en correspondance dans le paradigme est approximativement la même (i.e. sont statistiquement identiques). En d'autres termes, on aurait dû s'attendre à ce que la durée du [s] dans la structure \#sə soit à peu près identique à celle des structures $\mathrm{s} \# \mathrm{C}$, ce qui n'est pas le cas puisqu'il existe une différence statistiquement significative entre la durée de la fricative des deux structures. En effet, la durée de la fricative dans les formes \#sə est en moyenne plus longue de $8.38 \mathrm{~ms}$ que celle de la structure s\#C, ce qui, selon nous, provient du fait que la position syllabique de la fricative est différente dans le paradigme (attaque vs. coda par exemple).

\subsection{Représentations phonologiques}

Comme le note Scheer (2009:103) à propos de la représentation de l'objet qui nous intéresse ici, « [...] les groupes $\mathrm{s}+\mathrm{C}$ sont des animaux étranges que les phonologues ne comprennent pas ». Ce type de séquences a effectivement donné lieu à un nombre relativement important de travaux dans la littérature phonologique (cf. Goad 2011 pour une revue pertinente). L'une des difficultés avec ces «animaux étranges » est qu'ils ne suivent pas le Principe de Séquençage de Sonorité (Clements 1990) selon lequel la sonorité doit croître au sein d'une attaque complexe. Pour contourner ce problème - sans toutefois le 
résoudre -, plusieurs analyses ont ainsi été proposées. Steriade (1982) analyse par exemple la fricative /s/ des séquences $\#_{\mathrm{SC}}$ comme étant extrasyllabique ou extramétrique (2a), où $\mathrm{O}$ représente le constituant «attaque de syllabe». Van der Hulst (1984) et Goldsmith (1990) considèrent quant à eux que cette consonne est directement licenciée par le nœud syllabique (2b) ou par le mot prosodique (2c) respectivement (où $\sigma$ symbolise la syllabe et PWd représente le mot prosodique). Ces trois approches ont en commun l'idée selon laquelle la fricative coronale de ce type de séquences forme ce que l'on nomme un «appendice» puisqu'elle n'est associée à aucun constituant syllabique après l'application de l'algorithme de syllabation. Contrairement à l'approche traditionnelle de la syllabe, Kaye (1992), qui place ses travaux dans le cadre de la phonologie du gouvernement, considère ce type de séquence non pas comme une attaque branchante mais comme une coda initiale suivie d'une attaque (2d $)^{3}$. En effet, dans le cadre de la phonologie du gouvernement, les chaînes segmentales sont pleinement syllabées dans le lexique et, par conséquent, aucun matériel segmental ne peut être sous analysé par l'algorithme de syllabation (extrasyllacité) puisque celui-ci n'entretient aucune relation avec les règles phonologiques (cf. Scheer 2002 pour une discussion de la notion d'extrasyllabicité). A ce titre, nous pensons que la notion d'extrasyllabicité n'est qu'une manière d'exprimer ce que nous ne comprenons pas.
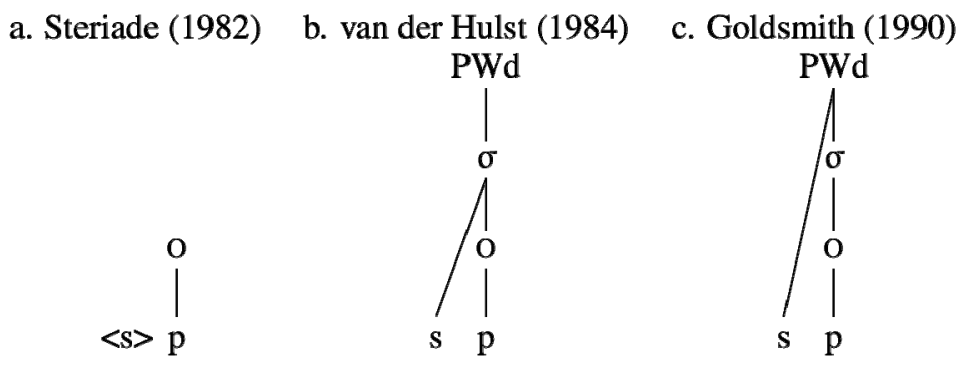

\section{d. Kaye (1992)}

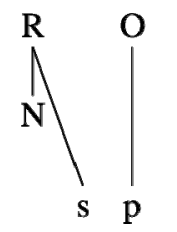

A l'instar de Kaye (1992), nous considérons que les séquences \#sC lexicales peuvent être structurellement analysées comme une coda initiale suivie par une attaque. Néanmoins, contrairement à Kaye (1992), nous plaçons notre analyse dans le cadre d'une approche strictement CV (Lowenstamm 1996, Scheer 2004 entre autres), une extension radicale de la phonologie du gouvernement. Dans cette approche, la structure prosodique est universellement composée d'une stricte consécution de $\mathrm{C}$ et de $\mathrm{V}$ (attaque-noyau), soit $\mathrm{C}_{1} \mathrm{~V}_{1} \mathrm{C}_{2} \mathrm{~V}_{2} \ldots \mathrm{C}_{n} \mathrm{~V}_{n}$. Contrairement à la représentation classique de la structure syllabique, qui considère les relations hiérarchiques de manière verticale par l'intermédiaire de constituants, la théorie $\mathrm{CV}$ fait appel à deux relations latérales (horizontales, droite-gauche) pour structurer la chaîne prosodique : le gouvernement et le licenciement. Cette manière d'appréhender la structure prosodique fait deux prédictions majeures : (i) la notion de coda en tant que constituant n'existe pas $^{4}$ et (ii) les constituants branchants sont illicites. Ainsi, dans un modèle strictement $\mathrm{CV}$, une coda est une consonne suivie par un noyau vide ( ), une géminée est un segment associé à deux positions $\mathrm{C}$ et une voyelle longue est un segment associé à deux positions $\mathrm{V}$ (où $\mathrm{A}=$ attaque et $\mathrm{N}=$ noyau) :

$$
\begin{aligned}
& \text { a. Syllabe fermée b. Consonne géminée c. Voyelle longue } \\
& \begin{array}{cccc}
A & N & A & N \\
C & \text { V } & C & \emptyset
\end{array} \\
& \underbrace{\mathrm{A}}_{\mathrm{C}} \mathrm{V}_{\mathrm{V}}^{\mathrm{N}} \\
& \left.\right|_{C} ^{A} \underbrace{A}_{V}{ }^{N}
\end{aligned}
$$

Sous cette hypothèse, et si nous prenons en considération le rôle actif du site CV initial en français (Lowenstamm 1999) ${ }^{5}$, la seule manière de représenter les séquences $\#_{\mathrm{sC}}$ lexicales consiste à associer la fricative /s/ «parasite » à la position $\mathrm{C}_{0}$ du site $\mathrm{CV}$ initial (Lowenstamm 2002). Dans la représentation de la forme /зроь/ sous (4), la position $\mathrm{V}_{0}$, proprement gouvernée (GP) par la position $\mathrm{V}_{1}$, peut rester non interprétée phonétiquement (cf. le Principe des Catégories Vides, Scheer 2004 entre autres) :

(4) Séquence \#sC lexicale 


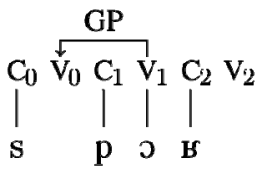

Si une coda est une consonne associée à une position $\mathrm{C}$ suivie d'une position $\mathrm{V}$ qui est vide, alors la fricative coronale /s/ des séquences $\#_{\mathrm{s}} \mathrm{C}$ est en position de «coda initiale », ce qui explique en partie la particularité de ces étranges objets que sont les séquences $\mathrm{AsC}$.

Contrairement aux séquences $\#_{\mathrm{s}} \mathrm{C}$ lexicales, où la fricative $/ \mathrm{s} /$ est associée à la position $\mathrm{C}_{0}$, nous supposons que la différence de durée observée dans les séquences $\mathrm{s}$ C dérivées de l'effacement du schwa découle d'une différence de structure prosodique. En effet, au moins deux stratégies peuvent émerger après l'effacement du schwa du démonstratif : (i) la fricative est resyllabée dans la « coda » de la syllabe précédente ou (ii) la fricative forme une syllabe en elle-même (consonne syllabique). Si nous devions envisager la première solution, nous n'aurions peut être pas trouvé de différence statistiquement significative de la durée de la fricative entre les séquences $\#_{\mathrm{s} C}$ et $\mathrm{s} \# \mathrm{C}$ puisque dans les deux cas cette consonne se trouve en position de «coda ». Sous cette hypothèse, il est néanmoins possible de considérer que la différence de durée de la fricative résulterait du locus de la «coda ». En d'autres termes, le fait que la coda soit en position initiale ou finale de mot aurait une influence sur la durée de la fricative des séquences $\mathrm{s}+\mathrm{C}$. Contrairement à la représentation sous (4), où la coda est en position initiale de mot, la forme donnée sous (5) illustre le cas où l'effacement du schwa laisse la fricative [s] en position de coda finale de mot :

(5) Séquence s\#C dérivée (version coda finale)
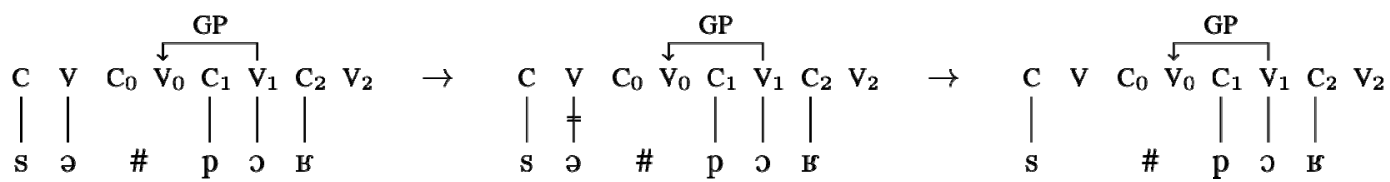

Dans ce type de représentation, le démonstratif ne peut se déplacer sur le site CV initial du mot suivant puisqu'il n'est pas en position de clitique. La différence de durée observée devrait par conséquent émerger de la structure prosodique.

La seconde solution consiste à postuler que la différence de durée qui existe entre la fricative coronale des deux types de clusters résulte de l'association de [s] à la position V laissée vacante après l'effacement du schwa :

(6) Séquence s\#C dérivée (version consonne syllabique)

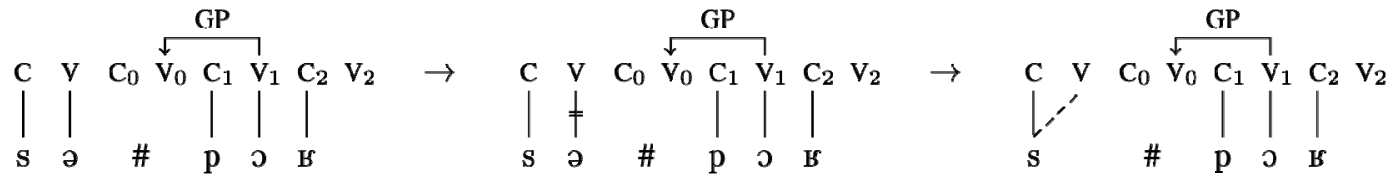

L'hypothèse de Rialland (1986) selon laquelle il n'y a pas de « resyllabation » de la consonne qui précède le schwa n'est pas falsifiée puisque il est tout à fait possible, dans un cadre strictement CV, d'envisager le fait que la consonne la plus sonante d'une séquence consonantique dérivée (dans notre cas la fricative coronale non voisée) puisse occuper une position à la fois consonantique et vocalique; configuration typique d'une consonne syllabique dans un tel cadre d'analyse (cf. Scheer 2004). Par ailleurs, les observations de Toft (2002:137) confirment le fait que les les consonnes syllabiques (de l'anglais) sont généralement plus longues que leurs contre-parties non syllabiques, ce qui va dans le sens de nos résultats. 


\section{Conclusion}

Les résultats de cette recherche sont tout à fait intéressants puisqu'ils mettent en lumière certains effets liés à l'effacement optionnel du schwa en français standard dans un contexte particulier. Ainsi, la fricative coronale des clusters dérivés de l'effacement du schwa est statistiquement plus longue que celle des clusters lexicaux alors que la durée des occlusives suivantes est à peu près identique. Selon nous, cette différence découle du fait que la fricative des clusters dérivés n'occupe pas la même position prosodique que la fricative des clusters lexicaux. Nous avons montré que dans un cadre strictement $\mathrm{CV}$, la fricative des séquences \#sC peut être analysée comme une coda initiale. En revanche, la fricative des séquences s\#C peut être analysée soit comme une coda finale de mot soit comme une consonne syllabique.

\section{Références bibliographiques}

Anderson, S. R. (1982). The analysis of french shwa: Or, how to get something for nothing. Language 58, 534-573.

Barnes, J. et Kavitskaya, D. (2003). Phonetic analogy and schwa deletion in french. In Larsen, J. et Paster, M. (éds.): Proceedings of the Twenty-Eighth Annual Meeting of the Berkeley Linguistics Society, 39-50, Berkeley, Berkeley Linguistics Society.

Byrd, D. (1993). 54,000 American stops. UCLA Working Papers in Phonetics 83, 97-116.

Clements, G. N. (1990). The role of the Sonority Cycle in Core Syllabification, in Kingston J. and Beckman M. (éds.), Laboratory Phonology 1: Between the Grammar and Physics of Speech, Cambridge University Press: Cambridge.

Dell, F. (1973). Les règles et les sons. Imprimerie Nouvelle, Orléans.

Fougeron, C. et Steriade, D. (1997). Does the deletion of french schwa lead to the neutralization of lexical distinctions? In Proceedings of the Eurospeech conference, 943-946, Rhodes.

Goad, H. (2011). The representation of sC clusters, In M. van Oostendorp, C. Ewen, E. Hume \& K. Rice (éds.). The Blackwell companion to phonology, 898-923, Oxford: Wiley-Blackwell.

Goldsmith, J. (1990). Autosegmental and metrical phonology. Oxford: Blackwell.

Harris, J. et Lindsey, G. (1995). The elements of phonological representation. In J. Durand \& F. Katamba (éds). Frontiers of phonology: atoms, structures, derivations. 34-79. Harlow, Essex: Longman.

Hulst, H. van der (1984). Syllable structure and stress in Dutch. Dordrecht: Foris.

Kaye, J., Lowenstamm J. et Vergnaud J.R. (1990). Constituent structure and government in phonology. Phonology 7, 193-231.

Kaye, J. (1992). Do you believe in magic? The story of s+C sequences. SOAS Working Papers in Linguistics 2, 293313.

Klatt, DH. (1976). Linguistics uses of segmental duration in English: acoustic and perceptual evidence. Journal of Acoutical Society of America 59, 1208-1221.

Lowenstamm, J. (2002). Paramétrisation du CV initial (le bébé et l'eau du bain), papier présenté aux 4èmes Journées Internationales du GDR CNRS 1954 «Phonologies », Université Stendhal, Grenoble, France.

Lowenstamm, J. (1996). CV as the only syllable type. In Durand J. et Laks, B., éditeurs : Current trends in phonology: models and methods, pages 419-441. European Studies Research Institute, Salford, Manchester.

Lowenstamm, J. (1999). The beginning of the word. In Rennison, J. et Kühnhammer, K. (éds.), Phonologica 1996, pages 153-166. Holland Academic Graphics, The Hague.

Nasukawa, K. \& Backley, P. (2008). Affrication as a performance device. Phonological Studies 11, 35-46. 
O'Shaughnessy, D. (1981). A study of French vowel and consonant durations. Journal of Phonetics 9, 385-406.

Rialland, A. (1986). Schwa et syllabes en français. In Wetzels, L. et Sezer, E. (éds.). Studies in Compensatory Lengthening, 187-226. Foris Publications, Dordrecht.

Scheer, T. (1998). A unified model of Proper Government. The Linguistic Review 15, 41-67.

Scheer, T. (2002). There is no extrasyllabicity. Poster présenté au 10th Manchester Phonology Meeting, 23-25 mai 2002.

Scheer, T. (2004). A lateral theory of phonology. Vol 1: What is CVCV, and why should it be ? Mouton de Gruyter, Berlin.

Scheer, T. (2009). Précis de structure syllabique. Lalies 29, 79-156.

Steriade, D. (1982). Greek prosodies and the nature of syllabification. Cambridge, MA: MIT dissertation.

Steriade, D. (2000). Paradigm uniformity and the phonetics-phonology boundary. In Broe, M. B. et Pierrehumbert, J. B. (éds). Papers in Laboratory Phonology V: Acquisition and the Lexicon, 313-335. Cambridge University Press, Cambridge.

Toft, Z. (2002). The phonetics and phonology of some syllabic consonants in southern british english. ZAS Papers in Linguistics 28, 111-144.

Tranel, B. (1995). Current issues in french phonology: liaison and position theories. In Goldsmith, J. A., (éd.). The Handbook of Phonological Theory, 798-816. Blackwell Publishers, Oxford.

Zue, V. W. (1976). Acoustic characteristics of stop consonants: a controlled study. Technical report 523, MIT Lincoln Laboratory:Lexiington (MA).

Il est intéressant de souligner le fait que O'Shaughnessy (1981:404) note que les occlusives des séquences \#sC sont moins longues de $26 \%$ que les occlusives en attaque simple (\#C).

2 Toutes choses égales par ailleurs, la durée du bruit de friction qui suit le relâchement des occlusives vélaires est généralement plus importante que celle des labiales et des coronales en vertu du fait que le dos de la langue est un articulateur moins véloce que les lèvres ou la couronne de la langue.

3 Pour des raisons liées à la notion de gouvernement inter-constituant, Kaye (1992) met en évidence le fait que la fricative des séquences $\mathrm{s}+\mathrm{C}$ doit être interprétée comme une coda dont l'attaque et le noyau sont vides par l'intermédiaire de ce qu'il nomme le « Magic Licencing ».

4

Dans ce type d'approche de l'organisation syllabique, une position de « coda » n'est rien d'autre qu'une consonne qui précède un noyau vide.

En français, ce site initial vide, qui correspond au début du mot (i.e. \# = CV), est requis pour l'analyse des clitiques et des séquences du type \#TR (où $\mathrm{T}=$ occlusives et $\mathrm{R}=$ sonantes) par exemple. 